\title{
Meta
}

Journal des traducteurs

Translators' Journal

\section{LAfARgA, F. y L. PegenAute (eds.) (2004) : Historia de la traducción en España, Salamanca, Editorial Ambos Mundos, 872 p.}

\section{Georges L. Bastin}

Volume 50, numéro 3, août 2005

Le prisme de l'histoire

The History Lens

URI : https://id.erudit.org/iderudit/011615ar

DOI : https://doi.org/10.7202/011615ar

Aller au sommaire du numéro

Éditeur(s)

Les Presses de l'Université de Montréal

ISSN

0026-0452 (imprimé)

1492-1421 (numérique)

Découvrir la revue

Citer ce compte rendu

Bastin, G. L. (2005). Compte rendu de [LAfARGA, F. y L. Pegenaute (eds.) (2004) : Historia de la traducción en España, Salamanca, Editorial Ambos Mundos, 872 p.] Meta, 50(3), 1053-1056. https://doi.org/10.7202/011615ar

Ce document est protégé par la loi sur le droit d'auteur. L'utilisation des services d'Érudit (y compris la reproduction) est assujettie à sa politique d'utilisation que vous pouvez consulter en ligne.

https://apropos.erudit.org/fr/usagers/politique-dutilisation/
Cet article est diffusé et préservé par Érudit.

Érudit est un consortium interuniversitaire sans but lucratif composé de l'Université de Montréal, l'Université Laval et l'Université du Québec à Montréal. Il a pour mission la promotion et la valorisation de la recherche. https://www.erudit.org/fr/ 
se penchent, toujours selon une approche contrastive et selon la théorie de l'argumentation de Anscombre et Ducrot, sur les concessives en français et en espagnol à partir d'un corpus constitué de Madame Bovary et de La Regenta, et de leurs traductions. Julia Pinilla, de son côté, étudie, dans un texte rédigé en français, la traduction des termes techniques au $\mathrm{XVIII}^{\mathrm{e}}$ siècle et en particulier les couples synonymiques. Finalement, E. Llácer Lorca et N. Estévez se tournent vers l'analyse de la fonction rythmique dans la traduction des Tales d'Edgar Allan Poe par Carlos Olivera (1884) pour y déceler des «zones d'inéquivalences phonétiques et syntaxiques».

Cet ouvrage, au-delà de la richesse et de l'abondance des données archéologiques historiographiques dans le contexte européen du $\mathrm{XvI}^{\mathrm{e}}$ au $\mathrm{XIX}^{\mathrm{e}}$ siècle, constitue un essai unique sur la méthodologie de l'historiographie traductologique. Il mériterait d'être traduit pour le plus grand bénéfice des historiographes traductologiques.

Georges L. Bastin

Université de Montréal, Montréal, Canada

\section{NOTES}

1. Lépinette, Brigitte (1997): La historia de la traducción. Metodología. Apuntes bibliográficos. Lynx, Vol. 14.

2. Op. cit.

3. Voir notre recension de López Alcalá, Samuel. La historia, la traducción y el control del pasado dans META 47-1, p. 132-134.

Lafarga, F. y L. Pegenaute (eds.) (2004): Historia de la traducción en España, Salamanca, Editorial Ambos Mundos, 872 p.

S'il existait encore le moindre doute quant au pays le plus prolifique en matière de publications en histoire de la traduction, il est désormais dissipé: c'est l'Espagne. L'ouvrage de Lafarga et Pegenaute en est un témoin éloquent: avec ses 872 pages, il vient couronner une production que l'on peut estimer, sans crainte d'exagération, à plusieurs milliers de pages sur le même sujet: l'histoire de la traduction en Espagne.

La tâche de rendre compte d'un tel «monument » apparaît dès lors comme une mission impossible. Pour un instant, nous avons pensé écrire cette recension de la manière dont Borges avait entrepris la traduction de l'Ulysse de Joyce, c'est-à-dire en ne le lisant pas et en n'en traduisant que les deux dernières pages! Soyons sérieux, mais pourquoi ne pas commencer ce compte rendu par la dernière page:

\section{Achevé d'imprimer sur les presses de l'imprimerie \\ CALATRAVA, dans la très culte cité de \\ Salamanca, le 30 septembre 2004, fête de SAINT JÉRÔME, docteur de l'Église, patron et protecteur des traducteurs}

Voilà qui «traduit» une planification éditoriale implacable ou, qui sait, un pieux mensonge. Quoi qu'il en soit, ce détail accompagne harmonieusement la couverture du livre qui arbore le relief de saint Jérôme dans la chapelle de l'Université de Salamanca.

Les directeurs du volume sont reconnus chez eux, ils n'en sont pas moins connus à l'étranger. Francisco Lafarga et Luis Pegenaute, professeur de philologie française à 
l'Universidad de Barcelona et professeur de traduction et d'interprétation à l'Universidad Pompeu Fabra de Barcelone respectivement, ont à leur actif une impressionnante production dont l'essentiel concerne l'histoire de la traduction. META a eu l'honneur et le plaisir d'accueillir le premier comme conférencier invité à l'occasion de son colloque anniversaire. Ils ont choisi, pour leur ouvrage monumental, la collection Biblioteca de Traducción de la maison d'édition Editorial Ambos Mundos de Salamanque, qui leur a concocté, en dépit de sa taille et de son poids, un volume très réussi.

L'ouvrage de Lafarga et Pegenaute est donc une Histoire de la traduction en Espagne. Il a été précédé par deux autres «histoires» espagnoles (Pym 2000 et Ruiz Casanova 2000). La première se présente comme un arrêt sur douze images, ou plutôt moments clés de l'histoire du monde hispanique depuis le $\mathrm{XII}^{\mathrm{e}}$ siècle: entre autres, l'École de Tolède, la traduction du Coran et de la Bible, Alphonse X, le castillan et l'Empire español, les traducteurs en exil, Ruben Darío, les anthologies de traduction poétique et les Jeux olympiques de Barcelone. La seconde offre un panorama historique selon la périodisation habituelle en littérature hispanique: le Moyen Âge, le Siècle d'Or, les XviII ${ }^{\mathrm{e}}, \mathrm{xIX}^{\mathrm{e}}$ et $\mathrm{xx}^{\mathrm{e}}$ siècles.

La présente histoire adopte une perspective historique-chronologique pour mettre en relation l'activité traduisante au cours des siècles et les poétiques de la traduction propres à chaque période depuis le $\mathrm{IV}^{\mathrm{e}}$ siècle jusqu'à nos jours. Quant à la périodisation, compte tenu que leur Histoire de la traduction est presque exclusivement celle de la traduction littéraire, les auteurs ont choisi celle généralement utilisée en historiographie de la littérature espagnole et demandé à des collaborateurs de premier ordre de se charger de chacune des périodes envisagées: le Moyen Âge (Julio-César Santoyo), la Renaissance et le Baroque (José María Micó), le xviII siècle ou de l'Illustration au Romantisme (Francisco Lafarga), la période romantique (Luis Pegenaute), le Réalisme et la fin de siècle (Luis Pegenaute), des Avantgardes à la guerre civile (Miguel Gallego Roca), de la guerre civile au passé récent (Miguel Ángel Vega), la situation actuelle (Luis Pegenaute). On remarquera, comme dans la plupart des histoires, que les périodes se rétrécissent à mesure que l'on se rapproche de l'époque actuelle.

Notons que ces différentes périodes font partie du premier chapitre: «La traduction dans l'aire de la culture castillane». Le second chapitre (il n'y en a pas d'autres) est consacré à la traduction dans d'autres aires linguistiques et culturelles. C'est peut-être là le plus grand mérite de cette Histoire: d'avoir placé aux côtés de la culture castillane les cultures catalane (Josep Pujol, Josep Solervicens, Enric Gallén et Marcel Ortín), la culture galicienne (Camiño Noia) et la culture basque (Xabier Mendiguren), pour ainsi donner de l'Espagne une vision la plus représentative possible. Il faut en effet reconnaître qu'il aurait été impossible d'ignorer la richesse de l'histoire de la traduction et des publications sur le sujet dans ces diverses aires linguistiques et culturelles d'Espagne.

On saluera dans cet ouvrage la capacité de coordination des directeurs qui, tout en laissant aux auteurs la liberté de traitement de leur sujet, ont su préserver la cohérence entre les différentes parties. Comme l'indiquent les directeurs de la collection, Román Álvarez et $\mathrm{M}^{\mathrm{a}}$ Carmen África Vidal, les auteurs ont su adopter les mêmes principes méthodologiques de base en tenant compte, chacun dans leur partie respective, de «la diversité des traductions, des rapports entre celles-ci et la pensée traductrice, de la réception de la littérature traduite et des liens entre la littérature traduite ou importée et la littérature locale»(p. II) (notre traduction).

L'index onomastique de près de 4000 noms traduit, si l'on peut dire, l'immensité de cette tâche historique. Certes, le travail archéologique à la base de la présente histoire n'est pas le fait des seuls collaborateurs de l'ouvrage. Comme nous le disions, les chercheurs espagnols ont produit des milliers de pages d'histoire, à commencer par les deux plus grands répertoires bibliographiques publiés en Espagne en 1996 par Julio-César Santoyo et Fernando Navarro. Il ne faudrait pas oublier non plus les textes «fondateurs» de Antonio Pellicer 
(1778) et Marcelino Menéndez Pelayo (1952-1953). La prouesse des différents auteurs dans la synthèse, et aussi l'interprétation, de ces répertoires et des travaux antérieurs. Un pas de géant vers une «histoire raisonnée» de la traduction.

Un simple coup d'œil à la table des matières permet d'apprécier, parallèlement à une démarche analytique typiquement littéraire - à laquelle il est normal de s'attendre quand la priorité est donnée à la traduction littéraire -, une approche sociologique plus empirique tournée vers la personne du traducteur et vers la vie et l'environnement des œuvres traduites. Voici quelques sous-titres très évocateurs: "Premiers témoignages, longs silences», "Quelques éléments quantitatifs», «Environnement culturel et social de la traduction», «Finalité de la traduction», «Rôle du traducteur», «L'avènement de la nouvelle école et la manie de la traduction», "Pensée: krausisme, positivisme, marxisme, irrationalisme», «Nouvelles maisons d'édition», "La science, la technique et les sciences appliquées», "La traductographie, cause et effet du changement social et politique», "Sociologie de la traduction: centres de formation, associations professionnelles, prix et revues», etc. Une approche historiographique traductologique donc et non seulement littéraire.

Certains lecteurs regretteront cette priorité absolue accordée au littéraire (dont on dit qu'il ne représente guère que $10 \%$ du marché... aujourd'hui), mais reconnaîtront que les références à la traduction religieuse, philosophique, scientifique et juridique ne manquent pas. Pour sa part, un sous-continent entier regrettera que les auteurs n'aient pas consacré ne fut-ce qu'un chapitre à l'Amérique hispanique. Ils auront repéré, avec plaisir, quelques références éparses à d'éminents traducteurs hispano-américains (Andrés Bello, Jorge Luis Borges, Ruben Darío, José Martí, Pablo Neruda, Juan Antonio Pérez Bonalde), mais c'est tout! La tâche, il faut bien l'avouer, aurait été bien malaisée et on comprend l'hésitation à se lancer dans pareille entreprise. Il est à espérer qu'une collaboration future porte fruit.

Une erreur s'est glissée dans la date de publication de Breve teoría de la traducción de Francisco Ayala. Cet opuscule, issu de quatre articles publiés en 1946 et 1947 par le supplément littéraire de La Nación de Buenos Aires, a bien été publié à Mexico par Obregón en 1956 et non pas en 1943 comme l'indique Miguel Ángel Vega (p. 568). Ainsi l'attestent, entre autres, le site Web de la Fundación Francisco Ayala (<www.us.es/ayala/vidacrono.htm $>$ consulté le 15-06-2005) et l'article de Sabio Pinilla et Fernández Sánchez reproduit dans le site HISTAL (<www.histal.umontreal.ca/espanol/versionsp.htm> consulté le 15-06-2005). Cette erreur, d'ailleurs répétée ailleurs (cela arrive souvent), comme beaucoup d'autres sont le lot des historiens. La rigueur nous commande de les éviter, mais la réalité en décide autrement. Qui, parmi nous, oserait affirmer n'en avoir jamais commis? Voyons-le ainsi: les erreurs sont là non pour accuser, mais pour être corrigées! D'autres doivent s'être glissées dans un ouvrage d'une telle envergure. Partons à leur recherche et corrigeons-les.

Aucune œuvre n'est parfaite, et les auteurs de celle-ci n'en ont jamais eu la prétention. Mais ils me semblent bien humbles par rapport au devoir accompli. Un devoir qui mérite d'être lu, connu et reconnu. On est en droit de se demander quel pays ou quelle région, après la prouesse espagnole, se lancera dans l'aventure. Le modèle est là, il suffit de s'en inspirer. Avis aux amateurs!

Cette recension n'aura pas rendu compte du contenu proprement dit de cette Histoire. Mission impossible? Non, mais aussi risquée, en quelques pages, que celle de consacrer un seul chapitre à l'Amérique hispanique... Aussi nous abstiendrons-nous; après tout, l'histoire, c'est de l'histoire, c'est des histoires. Il faut les lire, il faut les vivre. Celle que nous offrent Lafarga et Pegenaute figure parmi les plus belles.

Georges L. Bastin 


\section{RÉFÉRENCES}

Menéndez Pelayo, M. (1952-1953): Biblioteca de traductores españoles, Madrid-Santander, CSIC, vol. LIV-LVII de la Edición nacional de las obras completas.

Navarro Domínguez, F. (1996) : Manual de bibliografía española de traducción e interpretación. Diez años de historia: 1985-1995, Alicante, Universidad de Alicante.

Pellicer Saforcada, J. A. (1778): Ensayo de una biblioteca de traductores españoles, Madrid, Sancha.

Рум, A. (2000) : Negotiating the Frontier: Translators and Intercultures in Hispanic History, Manchester, St. Jerome Publishing.

Ruiz Casanova, J. F. (2000): Aproximación a una historia de la traducción en España, Madrid, Cátedra.

SAntoyo, J.-C. (1996) : Bibliografía de la traducción en español, catalán, gallego y vasco, León, Universidad de León.

Wyler, L. (2003): Línguas, poetas e bacharéis - Uma crônica da tradução no Brasil, Rio de Janeiro, Rocco, 158 p.

À l'instar des autres pays de l'Amérique latine, le Brésil a commencé depuis quelques années à se pencher sur son histoire de la traduction. Des auteurs comme Paulo Ronai (1987), Else Vieira Pires (1998), John Milton (1993 et 2001), Pagano et Vasconcelos (2003) et Campos (2004) ont en effet abordé le sujet. Le présent ouvrage de Lia Wyler vient toutefois combler un vide criant. Il est en effet le premier à embrasser l'intégralité de l'histoire brésilienne de la traduction, plus précisément de 1500 à 1979. Si l'auteure se limite à un bien bref panorama de la pratique traductrice dans ce pays, elle a l'immense mérite, en pionnière, d'ouvrir de nombreuses pistes de recherche. Rappelons toutefois, avant tout chose, que Lia Wyler est désormais internationalement connue et reconnue pour ses adaptations créatives de Harry Potter. Traductrice des cinq volumes jusqu'ici publiés, Lia Wyler a recréé le jargon haripotérien pour le plus grand plaisir des Brésiliens.

Contrairement à ce qu'indique son titre, ce livre est loin d'être une chronique, expression peut-être due à la modestie de l'auteure qui n'a vu dans son livre qu'un récit synthétique. Pourtant la minutie des données et la vision de l'ensemble font état d'une recherche en profondeur.

Cet ouvrage est un exemple de vulgarisation scientifique, une synthèse habile, rédigée dans un portugais impeccable et accessible, tant pour les chercheurs intéressés que pour les non-initiés. Que Wyler se réfère à la vie culturelle et politique brésilienne, ou qu'elle expose les grands événements qui ont marqué la pratique traduisante, elle donne à son texte une direction: survoler de façon claire et concise l'histoire de la traduction au Brésil, en guise d'introduction aux travaux qui ne devraient tarder dans ce domaine encore vierge. L'ouvrage aurait pu être intitulé: Esquisse d'une histoire de la traduction au Brésil.

Chez Wyler, le caractère informatif du texte permet aux lecteurs d'acquérir une connaissance de l'histoire du Brésil, nécessaire à la compréhension d'une perspective historique du domaine de la traduction et des horizons correspondants. L'auteure reconstitue, par exemple, la naissance et la consolidation de la profession de traducteur et d'interprète au Brésil, en abordant des sujets tels que le désintérêt des Portugais pour le développement intellectuel de la colonie, la dissémination de la langue tupi, l'établissement tardif de la première maison d'édition brésilienne (1808), la domination culturelle française et les différentes formes de censure.

L’ouvrage couvre la période qui va de la découverte du Brésil par les Portugais (1500) à la reconnaissance légale de la profession de traducteur (1979). Après une brève introduction, 